\title{
Correction to: Validation of a virtual intracorporeal suturing simulator
}

\author{
Yaoyu Fu ${ }^{1} \cdot$ Lora Cavuoto ${ }^{1}$ (D $\cdot$ Di Qi $^{2} \cdot$ Karthikeyan Panneerselvam $^{2} \cdot$ Gene Yang $^{4} \cdot$ Venkata Sreekanth Arikatla ${ }^{3}$. \\ Andinet Enquobahrie ${ }^{3} \cdot$ Suvranu De $^{2}$. Steven D. Schwaitzberg ${ }^{4}$
}

Published online: 5 December 2018

(c) Springer Science+Business Media, LLC, part of Springer Nature 2018

\section{Correction to: Surgical Endoscopy https://doi.org/10.1007/s00464-018-6531-3}

Disclosures Dr Schwaitzberg has no relevant conflicts related to this manuscript and is a consultant for Activ Surgical, Human Extensions, Arch Therapeutics, AcuityBio and $\mathrm{Nu}$ View Surgical. Yaoyu Fu and Drs. Lora Cavuoto, Di Qi, Karthikeyan Panneerselvam, Gene Yang, Venkata Sreekanth Arikatla, Andinet Enquobahrie, Suvranu De have no conflicts of interest or financial ties to disclose.

The surname of Sreekanth Arikatla incorrectly appeared as Sreekanth Artikala in original article.

In the Abstract, the values in the Results section were slightly incorrect and should read as follows:

Three questions from the face validity questionnaire were excluded due to lack of response. Ratings to 8 of the remaining 10 questions ( $80 \%$ ) averaged above a 3.0 out of 5. Average intracorporeal suturing completion time on the VBLaST-SS $\odot$ was 425 (standard deviation $=170 \mathrm{~s})$ seconds compared to $410(178 \mathrm{~s})$ seconds on the box trainer $(p=0.657)$. Deviation in needle insertion from the black dot was smaller for the

The original article can be found online at https://doi.org/10.1007/ s00464-018-6531-3.

Lora Cavuoto

loracavu@buffalo.edu

1 Department of Industrial and Systems Engineering, University at Buffalo, Buffalo, NY 14260, USA

2 Center for Modeling, Simulation and Imaging in Medicine (CeMSIM), Rensselaer Polytechnic Institute, Troy, NY, USA

3 Medical Computing Group, Kitware, Inc., Carrboro, NC, USA

4 Department of Surgery, University at Buffalo, Buffalo, NY, USA box trainer than the virtual simulator (1.69 vs. 7.10 , $p<0.001)$.

In the Results section, there was an incorrect reporting of the total number of medical students included. The first sentence should read as:

Nineteen medical students (MS, $n=19)$, six residents $(n=6)$ and a research assistant $(n=1)$ voluntarily enrolled in this study.

In the Results section on Face Validity, there was a mismatch between the data in the text and that reported in Table 2 for the highest rated question. The third sentence should read as:

Those questions that rated the highest were the usefulness of the VBLaST-SS $\odot$ simulation in learning handeye coordination skills compared to the FLS (3.88) and the degree of realism of the target objects in the VBLaST-SS $\odot$ compared to the FLS (3.84).

In the Results section on Performance, there were inaccuracies in the reported performance data. The Performance section paragraph should read as:

Performance.

Performance scores are shown in Table 3. Average intracorporeal suturing completion time on the VBLaST-SS $\odot$ was 425 (standard deviation $=170$ ) seconds compared to 410 (178) seconds on the box trainer $(p=0.657)$. Deviation in needle insertion from the black dot was smaller for the box trainer than the virtual simulator ( 1.69 vs. $7.10, p<0.001)$.

Accompanying this, Table 3 was incorrect as appeared, and should appear as:

As a result of the updated $p$ value, the fourth sentence in the Discussion should read as:

However, there was a trend toward significance for remaining incision gap and significant difference in 
Table 3 Comparison of the performance scores between VBLaST-SS@ and Real FLS

\begin{tabular}{lccr}
\hline & VBLaST-SS $\odot$ & Real FLS & $p$ value \\
\hline Completion time (s) (mean \pm SD) & $424.64 \pm 170.088$ & $409.68 \pm 178.240$ & 0.657 \\
Incision gap (mm) (mean \pm SD) & $1.139 \pm 0.873$ & $0.788 \pm 1.238$ & 0.064 \\
Deviations to black dots (mm) (mean \pm SD) & $7.102 \pm 4.944$ & $1.694 \pm 2.769$ & $<0.001$ \\
\hline
\end{tabular}

performance quality for remaining incision gap and needle insertion deviation. 